\title{
Can An Android Persuade You?
}

\author{
Kohei Ogawa, Christoph Bartneck, Daisuke Sakamoto \\ Takayuki Kanda, Member, IEEE, Tetsuo Ono, Hiroshi Ishiguro, Member, IEEE
}

\begin{abstract}
The first robotic copies of real humans have become available. They enable their users to be physically present in multiple locations at the same time. This study investigates what influence the embodiment of an agent has on its persuasiveness and its perceived personality. Is a robotic copy as persuasive as its human counterpart? Does it have the same personality? We performed an experiment in which the embodiment of the agent was the independent variable and the persuasiveness and perceived personality were the dependent measurement. The persuasive agent advertised a Bluetooth headset. The results show that an android is found to be as persuasive as a real human or a video recording of a real human. The personality of the participant had a considerable influence on the measurements. Participants that were more open to new experiences rated the persuasive agent lower on agreeableness and extroversion. They were also more willing to spend money on the advertised product.
\end{abstract}

\section{INTRODUCTION}

A great advantage of having a robotic copy of yourself is the ability to be physically present in two locations at the same time. In particular politicians might appreciate the ability to give two speeches at the same time during an election campaign. But while the physical appearances of androids have become almost indistinguishable from their human originals (see Figure 1), it is not clear to what degree the androids are able to convey the same personality and persuasive power as their human originals. Moreover, androids need to show a significant advantage to a screen character to justify the extra costs. A simple video transmission is currently easier and cheaper than a robotic copy, but some situations require a representation that is truly 3D. Human doppelgangers, for example, are frequently used to confuse paparazzi and terrorists. Robotic

Manuscript received February 11, 2009. This research was supported by the Ministry of Internal Affairs and Communications of Japan. The Geminoid HI-1 has been developed in the ATR Intelligent Robotics and Communications Laboratories.

K. Ogawa, T. Kanda, and H. Ishiguro are with ATR Intelligent Robotics and Communications Lab, 2-2-2 Hikaridai, Seikacho, Sorakugun, Kyoto 619-0288, Japan (e-mail: [ogawa, kanda, ishiguro]@atr.jp).

C. Bartneck is with the Department of Industrial Design, Eindhoven University of Technology, Den Dolech 2, 5600 MB Eindhoven, The Netherlands (phone: +31 (0)402475175; e-mail: c.bartneck@tue.nl).

T. Ono and K. Ogawa are with Future University-Hakodate, School of Systems Information Science, Department of Media Architecture, 116-2 Kamedanakano-cho, Hakodate 041-8655, Hokkaido, Japan (e-mail: tono@fun.ac.jp)

D. Sakamoto is JSPS Research Fellow, Graduate School of Information Science and Technology, The University of Tokyo, 7-3-1 Hongo, Bunkyoku, Tokyo 113-0033, Japan (e-mail: d.sakamoto@gmail.com) doppelganger could take over their owners place and ease some of the ethical difficulties associated with this dangerous business. It would matter much less if a robotic doppelganger would take a bullet than a human doppelganger. In this study we do not want to focus on the pure appearance of a robotic doppelganger, but on the persuasive power and personality androids may have. After all, it would be desirable if your robotic copy possesses the same persuasiveness and personality as yourself.

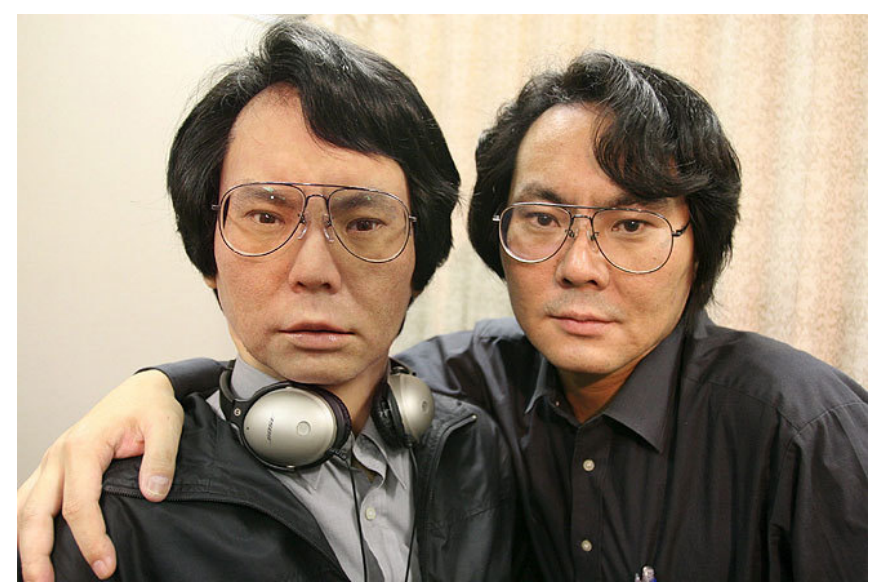

Figure 1:Geminiod HI-1 and Hiroshi Ishiguro

Persuasion can be defined as a social influence. It occurs when one person attempts to induce change in the beliefs, attitudes, or behavior of another person or a group of people [1]. Previous studies showed that the success of the persuasion depends on the source of the message [2, 3], the strength of the argument [4], and on the person being influenced $[2,5]$.

The persuasiveness of technology has become an important research field [6] and many robots are used in contexts where their main or primary purpose is to change the attitude, behavior or opinions of humans [7]. First studies on the persuasiveness of virtual characters and robots show promising results. Zanbaka et al. [1] compared the persuasiveness of virtual characters with the one of real humans by communicating the benefits of comprehensive exams to college students. They concluded that virtual characters are found to be as persuasive as real humans and that the realism of the character had no effect on its persuasive power. Shinozawa et al. used either a screen character or a robot to give recommendations to users. Their results showed that a robot's recommendation was stronger than that of a screen character [8]. Powers et al. compared people's response in a health interview to a screen agent and 
a robot [9]. They found only a few behavioral differences, but considerable differences in attitude. The participants spent more time with the collocated robots and had a more positive attitude. Kidd and Breazeal compared how users perceive a robot being in the same room as the user to a robot being shown on a screen [10]. They hypothesized that when the robot is physically present, it will be viewed as more persuasive than in the telepresent case. Their results showed that a robot is more engaging than an animated character and is perceived as more credible and informative, as well as being more enjoyable to interact with.

However, it is not clear to what degree androids may compare to their human originals in terms of persuasiveness and personality. In particular, what influence does the embodiment of a persuasive agent have on its persuasiveness and perceived personality. It has been shown that when the personality of the computer voice matches the users' personality: a) participants regarded the computer voice as more attractive, credible, and informative; b) participants were more likely to buy a product from the computer [11]. It is therefore necessary to not only measure the perceived personality of the persuasive agent, but also the personality of the participants. In summary, we are interested in the following research questions:

1. What influence does the embodiment of an agent have on its persuasiveness and perceived personality?

2. To what degree does the personality of the users influence their perception of the persuasiveness and personality of a persuasive agent?

\section{METHOD}

We performed a between-participant experiment in which the persuasive agent had three conditions. In the human condition Hiroshi Ishiguro presented a persuasive message, in the video condition a recording of Ishiguro's persuasive message was presented and in the android condition Geminoid HI-1 persuaded the audience. All three persuasive agents looked very much alike, which allowed us to focus on the embodiment of the agent, instead of its visual attractiveness.

\section{A. Measurements}

The participants' perceptions of the persuasive argument and message was assessed through a semantic differential questionnaire developed by Zanbaka, Goolkasian \& Hodges [1] which is based on previous work from Mullennix et al. [12].

Items related to perception of the argument and perception of the message was measured on a Likert-type scale. Items for each were as follows: perception of the argument (badgood; foolish-wise; negative-positive; beneficial-harmful; effective-ineffective; convincing-unconvincing); perception of the message (stimulating-boring, vague-specific, unsupported-supported, complex-simple, convincingunconvincing, uninteresting-interesting). Zanbaka et al. performed a principle components analysis on both concepts. The factor analysis of items related to the argument resulted in only one factor with a high reliability (Cronbach's alpha $=0.90$ ). The factor analysis of items related to the message resulted in two factors. The interesting factor (stimulating, specific, supported, convincing and interesting) accounted for 39 percent of the variance and the conservative factor, which explained 19 percent of variance. The Cronbach's alpha for the interesting factor was 0.76 . We translated the all the items to Japanese using the back-translation method.

In addition, we measured the persuasiveness of the speaker by asking the participants before and after the persuasive speech how much they would be willing to pay for the product. This repeated measure allowed us to compensate for individual differences. A certain participant, for example, might simply not like a given product. We calculated the variable price by subtracting the participants evaluation before seeing the agent from the on after seeing the agent. We will refer to this collection of questionnaires as the "persuasion questionnaire".

Several models and measurement tools have been proposed including the acknowledged Big Five Model [13], a brief version of the Big Five Model [14], Mowen's Personality Scale [15] and the established Myers-Briggs Type Indicator [16]. Many of these instruments consist of more than 100 items and can take up to one hour to complete. Since we are going to use several measurement instruments, it seems unreasonable to dedicate that much attention to only one tool. We therefore used the NEO FiveFactor Inventory (NEO-FFI) that only contains 60 items, which is designed to take only 15 minutes to fill in and which is available in the Japanese language. This questionnaire is a short version of the NEO PI-R instrument of the same author [17]. Albeit its briefness, this tool has demonstrated its validity and reliability. Ishiguro and the participants filled in this questionnaire about themselves.

The five factors in this personality questionnaire are neuroticism, extraversion, openness, agreeableness, and conscientiousness. Each of them is measured on a 0-48 scale. A person with a high neuroticism score can be described as "sensitive, emotion, and prone to experience feelings that are upsetting". A person with low neuroticism scale is "secure, hardy, and generally relaxed even under stressful conditions". A high extrovert score described a person as "Extrovert, outgoing, active, and high-spirited. You prefer to be around people most of the time" while a low score refers to "Introvert, reserved, and serious. You prefer to be alone or with a few close friends". "Open to new experiences. You have a broad interest and are very imaginative" describes a person with a high openness score and "Down-to-earth, practical traditional, and pretty much set in your ways." describes a person with a low openness score. A high agreeable score refers to "Compassionate, good-natured, and eager to cooperate and avoid conflict." and a low score to "Hardheaded, skeptical, proud, and competitive. You tend to express your anger directly". 
People with a high conscientiousness score are described as "Conscientious and well-organized. You have high standards and always strive to achieve your goals" while a people with a low score can be described as "Easygoing, not very well-organized, and sometimes careless. Your prefer not to make plans".

Unfortunately, the NEO-FFI version to rate another person has not yet been translated to Japanese. We therefore used the Japanese Property-Based Adjective Measurement questionnaire [18]. Its three components are highly correlated with the extraversion, openness and agreeableness components of the NEO-FFI (Hayashi, 1978.

The Geminoid HI-1 has received a considerable amount of media attention and hence it is possible that the participants might have seen or interacted with it. We therefore asked the participants if they have seen (e.g. television) the android or Ishiguro before (seen-agent), if they had met them (met-agent) or if they know them personally (know-agent). This allows us to take a possible bias into account in the statistical analysis.

In summary, we measured the persuasiveness of the presentation by its components argument, interesting and conservative. In addition, we calculated the change in the price estimation of the headset by subtracting the value from before the product presentation from the value after the presentation (price). We measured the personality of the participants and of Hiroshi Ishiguro with the NEO-FFI questionnaire. We measured the perceived personality of the persuasive agent with the Japanese Property-Based Adjective Measurement questionnaire. Finally, we measured the participants pre-knowledge of the android and Ishiguro.

\section{B. Setup}

We used the Geminoid-HI1 for this experiment since it allowed a direct comparison with his human equivalent, Hiroshi Ishiguro. The androids movement was based on motion data captured from Ishiguro performing the persuasive speech. The recording also included Ishiguro's voice, so that the lip movement of the android match the speech signal.

One limitation of the android is that it cannot grip and hold products or press small buttons reliably. We therefore decided to advertise a Bluetooth headset, since it can be demonstrated without having to handle it. Moreover, it may be assumed that a robot might be perceived to be more knowledgeable about electronic products than, for example, food products. The expertise of the speaker does have a considerable influence on his/her persuasiveness, which also holds true for the persuasiveness of machines [19, 20]. The headset was placed in the ear of the android and Ishiguro during the presentation.

A recording of Ishiguro performing the persuasive message was used in the video condition. For the recording, we placed a large television behind the camera that displayed the script of the persuasive message to make it easier for Ishiguro to remember the script. The same screen was placed behind the participants in the human condition. This procedure allowed Ishiguro to minimize the variations between his presentations. The video was projected onto a 110 by $175 \mathrm{~cm}$ screen, which approximates the actual size of Ishiguro and the android. The video had a resolution of 720 by 480 pixels. Figure 2 shows the experimental setup for the three conditions.

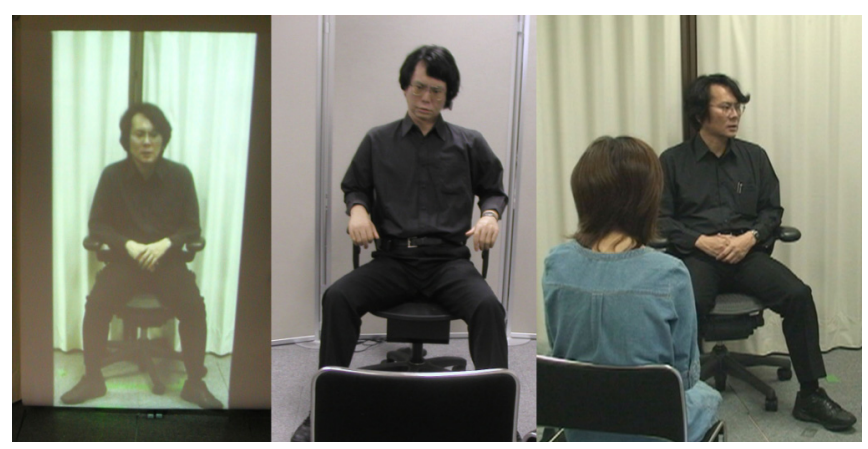

Figure 2: the three experimental condition: video (left), android (middle), and human (right)

The advertised headset did not contain any label or brand icon, so that the participants were not able to identify the headset. It was therefore impossible for the participants to simply know the price of the product.

\section{Procedure}

The participants joined the experiment in small groups. After welcoming the participants in room $\mathrm{A}$, the experimenter asked them to fill in an informed consent form. Next, the experimenter asked the participants to fill in a questionnaire that contained demographic questions and the NEO-FFI personality questionnaire. The participants were then asked how much they would pay for 30 products that were presented to them in a custom made catalogue. The products included furniture, electronic devices and accessories (see Figure 3).

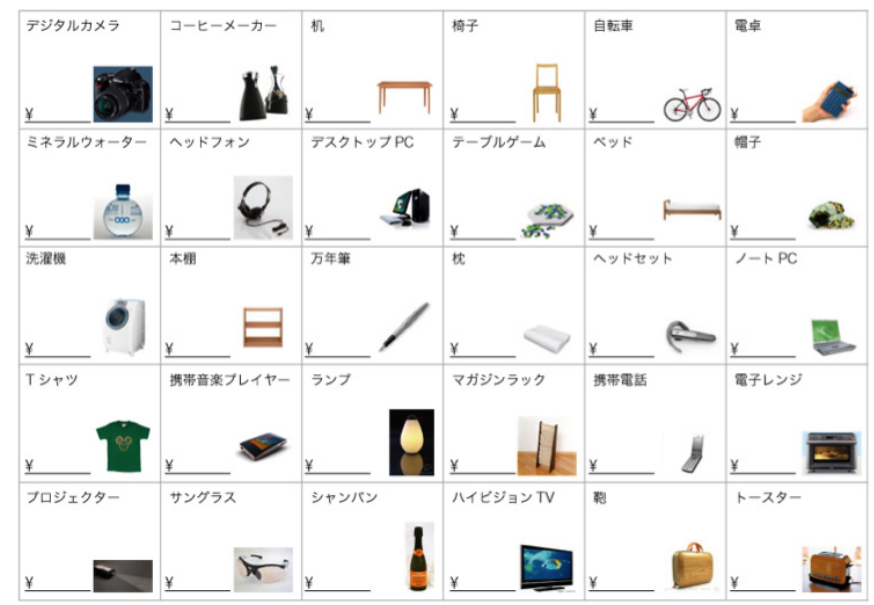

Figure 3: the product catalogue.

The experimenter then guided the participant into room B, where the persuasive agent (android, human, or the television screen) was located. The participants were seated 
on chairs that were arranged in a circle, 1 meter away from the persuasive agent (see Figure 2).

The experimenter left the room and the persuasive agent presented a Bluetooth headset. After the presentation was completed, the experimenter guided the participant back into room A where he/she filled in a questionnaire that contained the question how he/she was asked how much they would pay for the Bluetooth headset that was just presented to them, the persuasion questionnaire and the Japanese Property-Based Adjective Measurement questionnaire. In parallel to the experiment, we asked Ishiguro to fill in the personality questionnaire.

\section{Participants}

20 male and 12 female subjects participated in the study. They were between 19 and 25 years old (mean 21.1) and they received 3000 yen for their effort. The participants were recruited from a temporary work placement company called Arbeit Network, which specializes on the placement of students. All of the participants were students from a wide range of fields, including history, information science and psychology. 56 percent of the participants have never seen Ishiguro or the Geminoid HI-1 (e.g. television), 78 percent have never met them and 91 percent did not know them personally.

\section{RESUlTS}

A reliability analysis across the six arguments items resulted in a Cronbach's Alpha of 0.684, which is below the value of 0.90 reported in the original study of Zanbaka, Goolkasian \& Hodges [1]. The reliability of the interesting factor was 0.861 , which is above Zanbaka's value of 0.76 . The Cronbach's Alpha for the three components of the PropertyBased Adjective Measurement were 0.57 for openness, 0.86 for agreeableness, and 0.716 for extraversion. The reliability and validity estimations for the NEO-FFI are available from McCrae \& Costa [21].

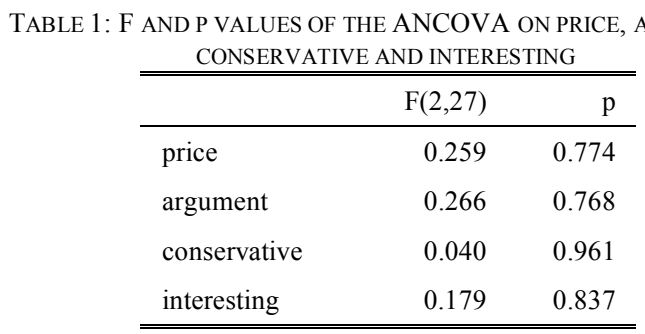

We performed an analysis of covariance (ANCOVA) in which the persuasive agent (human, video, android) was the independent variable. Seen-agent and gender were the covariants. Price, argument, interesting and conservative were the dependent variables. Neither covariant had a significant influence on the measurements. The persuasive agent also did not have a significant influence on the measurements (see Table 1).

We performed a second ANCOVA in which the persuasive agent was the independent variable. Seen-agent and the personality of the participant were the covariants.
The perceived extraversion, openness and agreeableness of the persuasive agent were the dependent variables. Notice that the Japanese Property-Based Adjective Measurement questionnaire does not have scales for the measurement of neuroticism or conscientiousness and therefore they do not appear in the further analysis.

Figure 4 shows the mean personality scores for all three conditions.

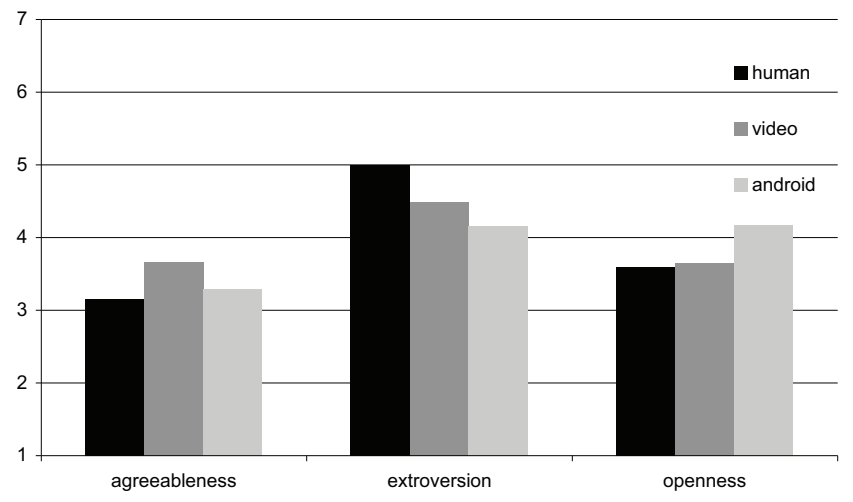

Figure 4: Mean personality scores for the persuasive agents

The persuasive agent did not have a significant influence on any of the measurements, although it did approach a significant level for openness $(\mathrm{F}(2,22)=2.567, \mathrm{p}=0.100)$. Post-hoc t-tests with Bonferroni corrected alpha showed that the human agent was rated almost significantly $(\mathrm{p}=0.153)$ less open (3.590) than the android agent (4.169). The covariant seen-agent and gender had no significant influence on the measurements. The personality of the participant also had an influence on the measurements. The openness of the participants had a significant influence on their rating on the extraversion of the agent $(\mathrm{F}(1,22)=8.700, \mathrm{p}=0.07)$.

TABLE 2: PEARSON CORRELATION BETWEEN THE OPENNESS OF THE PARTICIPANT AND THE PERSONALITY RATINGS FOR THE AGENT. (ITALICS INDICATE SIGNIFICANT CORRELATIONS AT P $<0.005$ )

\begin{tabular}{lrrr}
\hline \hline & $\begin{array}{r}\text { openness } \\
\text { participant }\end{array}$ & $\begin{array}{r}\text { agreeableness } \\
\text { agent }\end{array}$ & $\begin{array}{r}\text { openness } \\
\text { agent }\end{array}$ \\
\hline $\begin{array}{l}\text { agreeableness } \\
\text { agent } \\
\text { extraversion }\end{array}$ & -0.381 & & \\
$\begin{array}{l}\text { agent } \\
\text { openness agent }\end{array}$ & -0.389 & 0.323 & \\
\hline \hline
\end{tabular}

We performed a linear regression analysis to explore the relationship between the openness of the participants and their personality ratings of the agent. The openness of the participants was significantly correlated with the ratings for the agent on neuroticism, extraversion and openness (see Table 2). However, the personality ratings for the agent account for only 23.2 percent of the variance in the openness of the participant. Scatter plots revealed that the agreeableness and extroversion ratings for the agent decreased with rising openness of the participant.

Next, we performed a linear regression analysis between the participants' openness and the factors in the persuasive 
questionnaire (argument, interest, conservative and price). Only the price was significantly correlated with the openness of the participant $(r=3.57, p=0.022)$. A box plot revealed that the more participants are open to new experiences, the more they increase the amount they are willing to pay for the headset.

TABLE 3: MEAN SCORES FOR ISHIGURO'S SELF EVALUATION, MEAN SCORE FOR AGENT, T AND P VALUES FOR EXTRAVERSION, OPENNESS AND AGREEABLENESS

\begin{tabular}{lrrrr}
\hline & $\begin{array}{r}\text { mean self } \\
\text { score }\end{array}$ & $\begin{array}{r}\text { mean agent } \\
\text { score }\end{array}$ & $\mathrm{t}$ & $\mathrm{p}$ \\
\hline extraversion & 3.64 & 4.523 & 4.877 & 0.001 \\
openness & 6.41 & 3.828 & -26.194 & 0.001 \\
agreeableness & 3.50 & 3.367 & -0.945 & 0.352 \\
\hline
\end{tabular}

Last, we were interested to what degree the participants' evaluation of the agent's personality match with the score that Ishiguro gave himself. We divided the scores from the 48 -point scale of NEO-FFI questionnaire by $48 / 7=6.85$ to be able to compare the scores to the 7-point scale of the Property-Based Adjective Measurement. Table 3 shows the mean scores of Ishiguro and the participants. We then performed three one-sample t-test against the corresponding value from Ishiguro's questionnaire. The ratings for extraversion and openness were significantly different and mean scores from the Japanese Property-Based Adjective Measurement questionnaire hovered slightly around the center of the scale.

\section{DISCUSSION AND CONCLUSIONS}

The focus of this study was on the effects of the embodiment of the persuasive agent. Embodiment refers to the visual and haptic representation of the agent and not on the agents' voice. The same human voice was used in all conditions. Other studies explicitly focused on the influence on the agents' voice [12, 22].

Zanbaka et al. [1] had previously shown that college students found a virtual character as persuasive as a real human being. Their result was inline with other studies that showed that virtual characters are often treated similar to real humans $[23,24]$. We extend their results by concluding that a robotic copy of a real human is found to be as persuasive as its human original. Androids can therefore be considered as an alternative for presenting persuasive messages.

We also observed that embodiment of the agent might influence its perceived openness. The android was perceived as more open than its human or robotic counterpart. This might give the android a slight advantage over the video agent and justify the extra expense. We hope that if the number of participants increases, the effect would become significant.

Despite the considerable media attention that Ishiguro and his android received, it did not seem to have influenced the participants. Seeing a report on television might still be a different experience from standing in front of the real
McCoy.

Overall, the results suggest that the openness of the participants may play an important role in how the participants perceived the personality of the agent. The openness was negatively correlated with the agreeableness and extroversion ratings for the agent. Participants that were open also increased their willingness to spend money on the advertised headset.

However, the personality ratings that the agent received do not completely match the rating that Ishiguro gave himself. The short interaction time with the agent might not have been sufficient for the participants to better understand the agent. Ishiguro's great openness to new experiences may not communicate in the context of an advertisement. It remains a pleasant necessity to slowly get to know people and androids.

Besides the doppelganger scenario described in the introduction we can also envision another application domain for persuasive androids: advertisement. The androids could be used as sales agents in supermarkets and many other stores. Already today, audio and video messages are being used to persuade customers to purchase certain goods and first studies on the effectiveness of virtual agents are becoming available [25].

\section{A. Limitations and future work}

The results of our study are limited to the androids used in this study and the results may not generalize to other robots. Further research is necessary to determine in more detail what aspects of the embodiment contribute to the persuasiveness and personality of an android. We were also limited by the physical limitation of the Geminoid HI-1. It cannot move as smoothly as humans and it is not yet able to grasp objects. Future androids might have much better abilities and might therefore become even more persuasive.

Another drawback of this study was the limited number and diversity of the participants. To achieve more generalizable results, this study should be extended with a more diverse sample, in particular with more participants that are not university students. All the participants were Japanese and it has been shown that the cultural background of the users has an influence on their perception of a robot $[26,27]$. It would therefore be an interesting research question to repeat this experiment with users from other cultures.

\section{ACKNOWLEDGEMENT}

This work was partially supported by Grant-in Aid for Scientific Research (S), KAKENHI (20220002).

\section{REFERENCES}

[1] C. Zanbaka, P. Goolkasian, and L. Hodges, "Can a virtual cat persuade you?: the role of gender and realism in speaker persuasiveness," in SIGCHI conference on Human Factors in computing systems, Montreal, Quebec, Canada, 2006.

[2] L. L. Carli, "Gender, language, and influence," Journal of Personality and Social Psychology, vol. 59, pp. 941-951, 1990. 
[3] J. R. Priester and R. E. Petty, "Source Attributions and Persuasion Perceived Honesty as a Determinant of Message Scrutiny," Personality and Social Psychology Bulletin, vol. 21, pp. 637-654, June 1, 1995 1995.

[4] R. E. Petty and J. T. Cacioppo, Attitudes and persuasion - classic and contemporary approaches. Dubuque, Iowa: W.C. Brown Co. Publishers, 1981.

[5] C. P. Haugtvedt and R. E. Petty, "Personality and persuasion: Need for cognition moderates the persistence and resistance of attitude changes," Journal of Personality and Social Psychology, vol. 63, pp. 308-319, 1992.

[6] B. J. Fogg, Persuasive technology : using computers to change what we think and do. Amsterdam ; Boston: Morgan Kaufmann Publishers, 2003.

[7] K. Dautenhahn, "Roles and functions of robots in human society: implications from research in autism therapy," Robotica, vol. 21, pp 443-452, 2003.

[8] K. Shinozawa, F. Naya, J. Yamato, and K. Kogure, "Differences in effect of robot and screen agent recommendations on human decisionmaking," International Journal of Human-Computer Studies, vol. 62 , pp. 267-279, 2005.

[9] A. Powers, S. Kiesler, S. Fussell, and C. Torrey, "Comparing a computer agent with a humanoid robot," in Proceedings of the ACM/IEEE international conference on Human-robot interaction, Arlington, Virginia, USA, 2007, pp. 145-152.

[10]C. D. Kidd and C. Breazeal, "Effect of a robot on user perceptions," in Intelligent Robots and Systems, 2004. (IROS 2004). Proceedings. 2004 IEEE/RSJ International Conference on, 2004, pp. 3559-3564 vol.4.

[11]C. Nass and K. M. Lee, "Does computer-generated speech manifest personality? an experimental test of similarity-attraction," in SIGCHI conference on Human factors in computing systems, The Hague, The Netherlands, 2000, pp. 329 - 336.

[12]J. W. Mullennix, S. E. Stern, S. J. Wilson, and C. 1. Dyson, "Social perception of male and female computer synthesized speech," Computers in Human Behavior, vol. 19, pp. 407-424, 2003.

[13]L. R. Goldberg, "The development of markers for the Big-Five factor structure," Psychological Assessment, vol. 4, pp. 26-42, 1992.

[14]G. Saucier, "Mini-Markers: A Brief Version of Goldberg's Unipolar Big-Five Markers," Journal of Personality Assessment, vol. 63, pp. 506-516, 1994.

[15]J. C. Mowen, The $3 m$ Model of Motivation and Personality: Theory and Empirical Applications to Consumer Behavior. Boston: Kluwer Academic Publisher, 2000.

[16]C. G. Jung, Psychological types : or The psychology of individuation / by C. G. Jung. Translated by H. Godwin Baynes. London: Routledge and K. Paul, 1923.

[17]P. T. Costa and R. R. McCrae, "Normal personality assessment in clinical practice: The NEO Personality Inventory," Psychological Assessment, vol. 4, pp. 5-13, 1992.

[18]F. Hayashi, "The Fundamental Dimensions Of Interpersonal Cognitive Structure," Nagoya University, School of Education, Department of Education Psychology Bulletin, vol. 25, pp. 233-247, 1978.

[19]B. J. Fogg and T. Hsiang, "The elements of computer credibility," in SIGCHI conference on Human factors in computing systems, Pittsburgh, 1999, pp. 80-87.

[20]C. Bartneck, "How convincing is Mr. Data's smile: Affective expressions of machines," User Modeling and User-Adapted Interaction, vol. 11, pp. 279-295, 20012001.

[21]R. R. McCrae and P. T. Costa, "Validation of a five-factor model or personality across instruments and observers," Journal of personality and social psychology, vol. 52, pp. 81-90, 1987.

[22]S. E. Stern, J. W. Mullennix, C.-1. Dyson, and S. J. Wilson, "The Persuasiveness of Synthetic Speech versus Human Speech," Human Factors: The Journal of the Human Factors and Ergonomics Society, vol. 41, pp. 588-595, 1999.

[23]M. Slater and M. Usoh, "Body Centered Interaction in Immersive Virtual Environments," in Artificial life and virtual reality, N. Magnenat-Thalmann and D. Thalmann, Eds. New York: Wiley, 1994, pp. 125-148.
[24]D.-P. Pertaub, M. Slater, and C. Barker, "An Experiment on Public Speaking Anxiety in Response to Three Different Types of Virtual Audience," Presence: Teleoperators \& Virtual Environments, vol. 11, pp. 68-78, 2002.

[25]S. V. Suzuki and S. Yamada, "Persuasion through overheard communication by life-like agents," in IEEE/WIC/ACM International Conference on Intelligent Agent Technology, 2004. (IAT 2004), Beijing, 2004, pp. 225-231.

[26]C. Bartneck, T. Suzuki, T. Kanda, and T. Nomura, "The Influence of People's Culture and Prior Experiences with Aibo on their Attitude Towards Robots," AI \& Society - The Journal of Human-Centred Systems, vol. 21, pp. 217-230, 2007.

[27]C. Bartneck, "Who like androids more: Japanese or US Americans?," in 17th IEEE International Symposium on Robot and Human Interactive Communication, RO-MAN 2008, München, 2008, pp. 553-557. 